\title{
Description of Rotation-Invariant Textures using Local Binary Pattern Features
}

\author{
Prashant H. Gutte \\ PG student, \\ Dept. of Information Technology, \\ Walchand College of Engineering, Sangli, India
}

\author{
Prashant K Kharat \\ Assistant Professor \\ Dept. of Information Technology, \\ Walchand College of Engineering, Sangli, India
}

\begin{abstract}
Texture classification is one of the most interesting research topics in the field of computer vision. This paper aims at classifying static as well as dynamic textures (DT). Uniform Local binary pattern (LBP) is a combination of structural and statistical analysis model for classification of both static and dynamic textures. The LBP Histogram Fourier (LBP-HF) uses Fast Fourier Transform (FFT) for calculating global rotation invariant of LBP histogram for static texture classification. For dynamic texture classification, LBP-TOP method is used, which computes LBP from three orthogonal planes. It combines both motion and appearance together for classification. The results show that, LBP-HF outperforms uniform LBP as well as basic rotation invariant LBP method for static-textures and LBP-TOP gives higher accuracy for dynamic-textures.
\end{abstract}

\section{Keywords}

Texture, Local Binary Pattern, LBP-TOP, FFT

\section{INTRODUCTION}

Nature is rich in textures the surface of any visible object is textured at certain scale. A wealth of textures is observed on wood, plants, materials and skin. In a general sense, the word texture refers to surface characteristics and appearance of an object given by the size, shape, density, arrangement, proportion of its elementary parts. Texture based problems are very much popular in the field of image processing and computer vision. A texture with only appearance is known as static texture or simply an image and a texture with appearance along with motion is known as dynamic texture. Texture classification is widely used in various applications such as Geographical Information System, biomedical image analysis, Object Detection and content based image retrieval etc.

For static textures, features which are independent of angle to input texture image is known as rotation- invariant features. Statistical and Model based methods are two broad ways for classification. We have used combination of these two by using LBP. Recently LBP method is widely accepted in field of Computer vision, Pattern recognition etc. because of its simplicity, robustness to illumination and less compute intensive property.

Dynamic textures are sequences of images of moving scenes that exhibit certain stationary properties in spatiotemporal domain; these include sea-waves, smoke, foliage, water current etc. Recently, two spatiotemporal operators based on LBPs have been proposed for dynamic-texture description, i.e., volume LBPs (VLBP) [3] and LBP histograms from three orthogonal planes (LBP-TOP), which are XY, XT and YT planes. These operators combine motion and appearance together, and are robust to translation and illumination variations. The LBP-TOP has performed very well in different types of computer vision problems, such as dynamic-texture recognition, segmentation and synthesis, Facial-expression recognition, visual speech recognition, activity recognition, and analysis of facial paralysis.

In this paper for static textures, we first calculate the uniform LBP histogram from an image and then by applying FFT we get LBP-HF. FFT is used for faster calculation over DFT. The proposed LBP-HF features differ from the previous versions of rotation-invariant LBP since the LBP-HF features are computed from the histogram representing the whole region, i.e., the invariants are constructed from the histogram of noninvariant LBPs instead of computing invariant features independently at each pixel location. This idea is then extended to the spatiotemporal domain by using LBP-TOP method.

\section{ROTATION-INVARIANT STATIC TEXTURES}

\subsection{Basic LBP Method}

Local binary pattern method is introduced by Ojala et. al in 2000 [1]. In last decade there were various applications which are based on Local Binary Pattern method such as face recognition, Activity recognition etc. LBP method is easier to understand and less compute intensive makes it useable widely. Apart from this LBP is inherently robust to monotonic grey level changes, adds a different strength into it. For characterize the spatial structure of the local image texture, LBP code is calculated by comparing circular neighboring pixel. To do this, a circular neighborhood denoted by $(P, R)$ is defined. Here $P$ represents the number of sampling points and $R$ is the radius of neighborhood shown in Figure.1. These sampling points around pixel $(X, Y)$ lie at co-ordinates $\left(X_{p}\right.$, $Y_{p}$ ) given by

$$
\begin{aligned}
X_{p} & =X+R \cos (2 \pi p / P) \\
Y_{p} & =Y-R \sin (2 \pi p / P)
\end{aligned}
$$

LBP label for the center pixel $(X, Y)$ of image $f(X, Y)$ is obtained through

$L B P_{P, R}(X, Y)=\sum_{p=0}^{p-1} S\left(f(X, Y)-f\left(X_{p}, Y_{p}\right)\right) 2^{p}$

Where, $S(z)$ is the thresholding function

$$
S(z)=\left\{\begin{array}{cl}
1, z>=0 \\
0, & , z<0
\end{array}\right.
$$




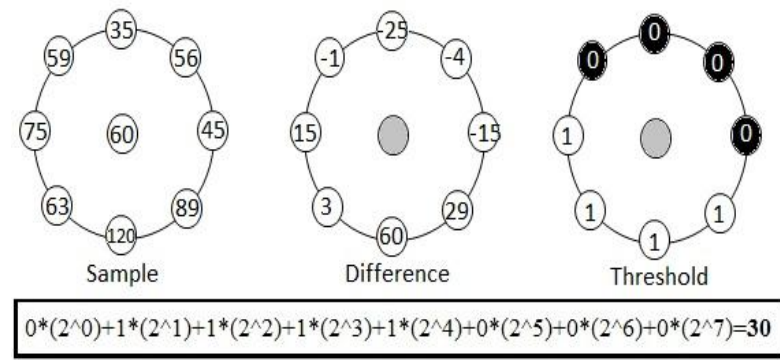

LBP Value $=\mathbf{3 0}$

Figure 1: Circular sampling of neighbor pixels points $(P)$ with radius $(R)$ i.e. $(P, R) \&$ calculation of $L B P$ for $3 \times 3$ block.

Here, Figure. 1 shows the calculation of local binary pattern value from the $(8,1)$ neighborhood considering center pixel as threshold. Further we consider only uniform patterns[4] to calculate LBP histogram. Uniform patterns are the patterns which has at most two shifts from 1 to 0 or 0 to 1 .

\subsection{LBP-HF for Static Structures}

The original rotation-invariant (RL- LBP) operator based on uniform patterns RI-LBP is achieved by circularly rotating each bit pattern to the minimum value. For example, the bit sequences 11000011, 11100001, and 01111000 arise from different rotations of the same local pattern, and they all correspond to the normalized sequence 00001111. This means that all the patterns from one row of bins [2] are replaced with a single label.

Here we have used Fast Fourier Transform to calculate global features from uniform LBP histogram instated of calculating invariant at each pixel independently. This makes RI-LBP feature set sub set of LBP-HF.

Based on the property, which states that rotations induce shift in the polar representation $(P, R)$ of the neighborhood, LBPHF gives a class of features that are invariant to the rotation of the input image, namely, such features computed along the input histogram rows, which are invariant to cyclic shifts. FFT is applied to LBP histogram as

$$
H(n, u)=\sum_{r=0}^{P-1} h_{I}\left(U_{p}(n, r)\right) e^{-i 2 \pi u r / P}
$$

Where, $h_{I}\left(U_{p}(n, r)\right)$ is the specific uniform LBP histogram pattern. Generation of LBP-HF features fallows following flow diagram.

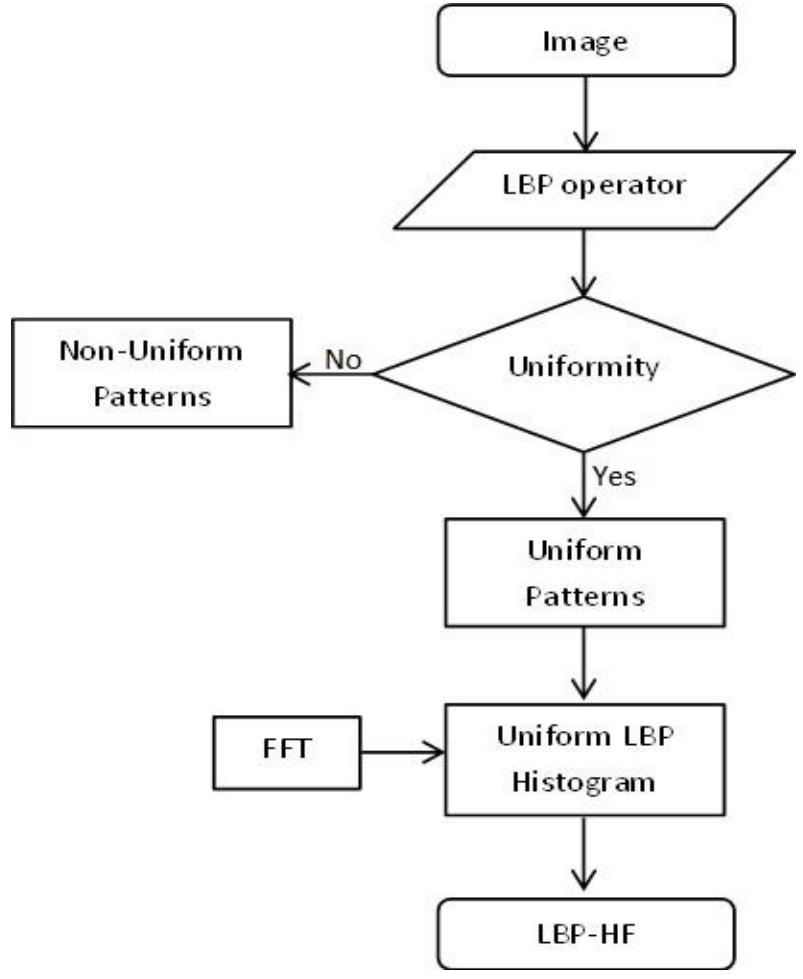

Figure 2: Flow diagram for generation of LBP-HF features

\subsection{Results for Static Structures}

Here we have used OUTEX_TC_00012 Data Set which contains total 9120 images of 24 different classes. Out of which 480 images are used for generating training features i.e. 20 images per class, remaining 8640 images are divided into two test case i.e. each class contains 180 images of 24 different classes.

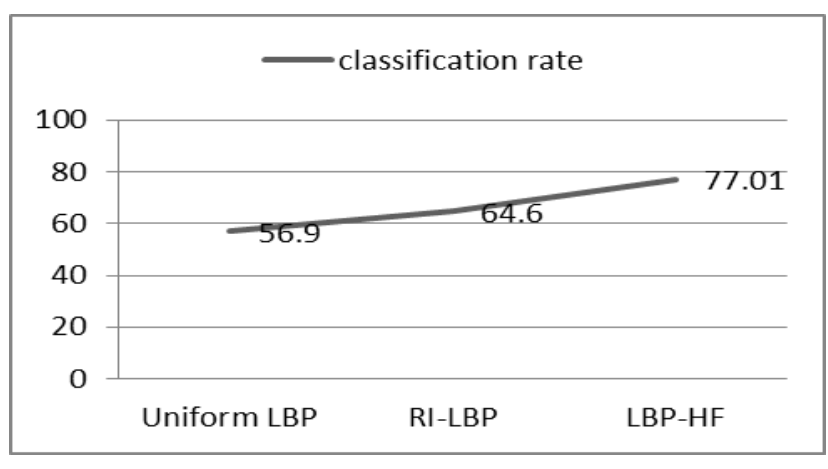

Figure 3: Texture classification rates on OUTEX_TC_00012 data set with $(8,1)$ neighborhood.

Results shows better classification rate for LBP-HF over uniform LBP and RI-LBP. Figure 3. Shows classification results for our popsed method out perforemed other existing LBP based methods.

\section{ROTATION INVARIANT DYNAMIC TEXTURES}

\subsection{LBP-TOP for Dynamic Textures}

In last part we have constructed LBP-HF features for rotation invariant static textures classification and obtained significantly better result as compare to other existing LBP 
methods. Now in this part we extend same idea in space and time i.e. in Dynamic textures (DT). Here DT recognition is utilized as a case study. DT is used for motion analysis. The recognition and the segmentation of DTs have attracted growing interest in recent years.

Here we use Three Orthogonal plane concept with LBP-HF, where XY plane gives appearance of the Dynamic texture and $\mathrm{XT} \&$ YT gives motion of the Dynamic texture. We consider different planes for calculation of LBP value. Figure. 4 shows the three planes which calculate the LBP from three different planes and concatenated to form a single LBP histogram.

The radii in axes $\mathrm{X}, \mathrm{Y}$, and $\mathrm{T}$ and the number of neighboring points in the XY, XT, and YT planes can also be different, which can be marked as $R_{X}, R_{Y}$ and $R_{T}, P_{X Y}, P_{X T}$, and $P_{Y T}$ respectively. The corresponding feature is denoted as LBP$\operatorname{TOP}\left(P_{X Y}, P_{X T}, P_{Y T} ; R_{X}, R_{Y}, R_{T}\right)$.

Suppose the coordinates of the center pixel are $\left(x_{c}, y_{c}, t c\right)$ the coordinates of neighborhood pixels in:

$\mathrm{XY}$ plane is given by

$x \_p=x_{-} c-R_{-} X \sin \left(2 \pi p / P_{-} X Y\right)$

$y_{-} p=y_{-} c-R_{-} Y \operatorname{coss}\left(2 \pi p / P_{-} X Y\right)$

$t_{p}=t_{c}$

Similarly for XT plane

$x_{-} p=x_{-} c-R_{-} X \sin \left(2 \pi p / P_{-} X T\right)$

$y_{p}=y_{c}$

$t_{-} p=t_{-} c-R_{-} T \cos \left(2 \pi p / P_{-} X T\right)$

Similarly for YT plane

$x_{p}=x_{c}$

$y_{-} p=y_{-} c-R_{-} Y \cos \left(2 \pi p / P_{-} Y T\right)$

$t_{-} p=t_{-} c-R_{-} T \sin \left(2 \pi p / P_{-} Y T\right)$

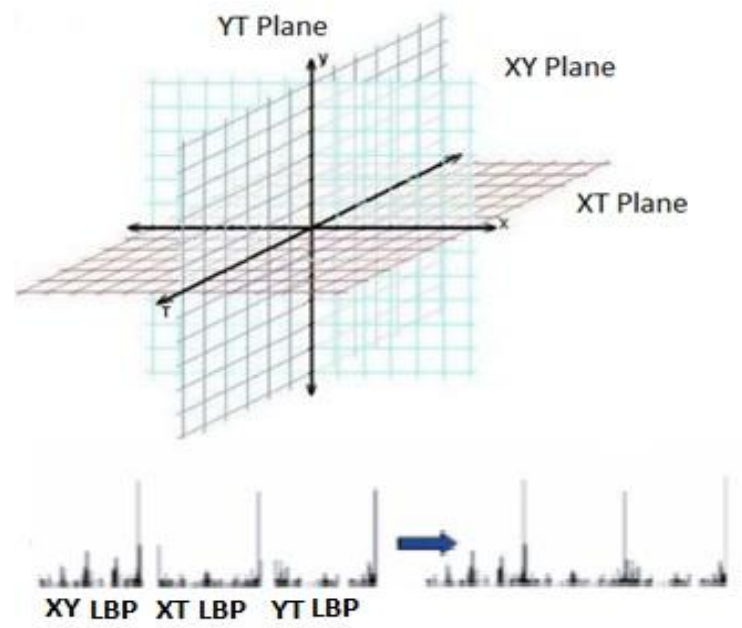

Figure 4: LBP-TOP Representation
This is different from the ordinary LBP widely used in many papers and it extends the definition of LBP.

For rotation invariant in DT we consider only two planes i.e. either XY along with XT, or XY along with YT for analysis because, rotation of either planes (i.e. XT and YT) with $90^{\circ}$ doesn't signify anything more, rather they are same after rotation of $90^{\circ}$.

After calculation of LBP's we apply 1-D FFT on each plane separately to get HFLBP-TOP feature. Figure 4 shows the flow diagram for generation of feature vector. The feature vectors generated by this method are significantly smaller than the feature vector generated by VLBP method [3]. Results shows HFLBP-TOP shows good recognition with different neighborhoods over VLBP. HFLBP-TOP uses flowing flow diagram for generation of feature vector:

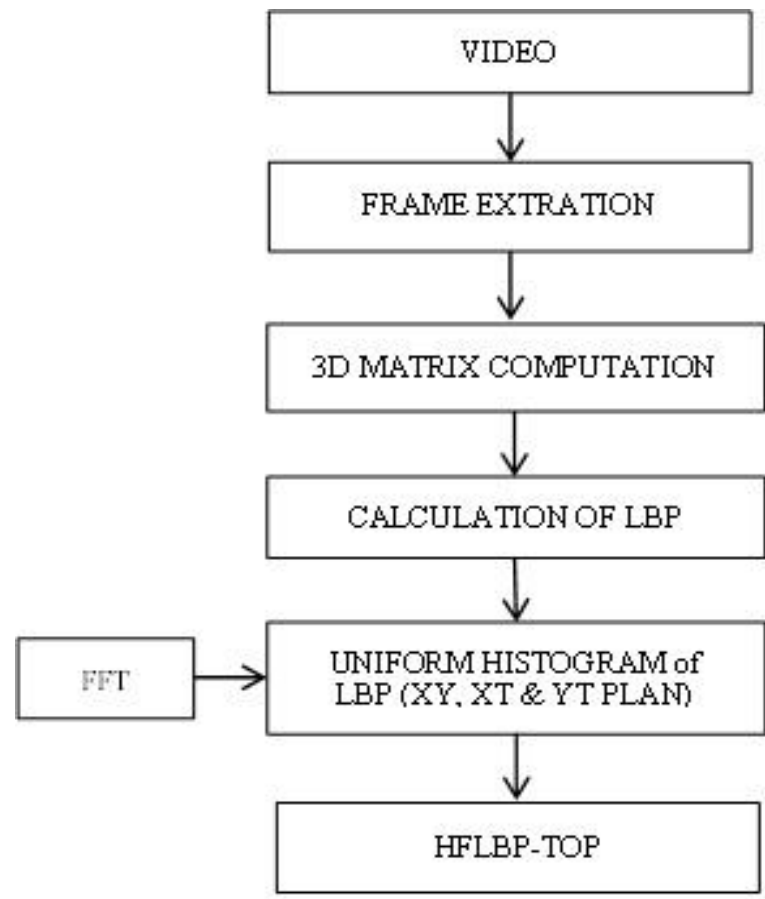

Figure 5: Flow diagram for generation of HFLBP-TOP features

\subsection{Results for Dynamic Textures}

Here we have used 8 different texture video to generate data set from DynTex Data set. Each video is of $10 \mathrm{sec}$ with 25 frames per second. We extracted frame from each video and generated five different textured videos having some rotation with equal number of frames i.e. of 50 frames per video. For experiment we consider all the video frames are of same size (i.e. Height and width) of all the frames are equal.

As we are considering only two planes at a time (i.e. XY \& $\mathrm{XT}$ or XY \& YT) the histogram plot for HFLBP-TOP is as shown in Figure 6.

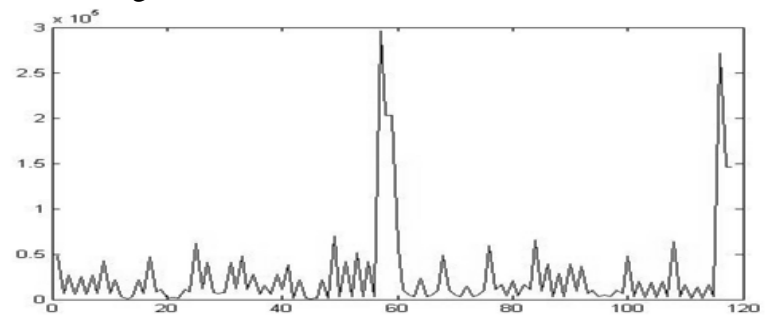

Figure 6:1-D HFLBP-TOP plot for "water current" 
HFLBP-TOP [2] performed well in comparison to VLBP, as VLBP features considers only four neighbors which itself forms too bigger feature vector as that of LBP-TOP. Here we computed LBP-TOP with (i.e. LBP-TOP L,8,1,1 ) eight neighborhood points with radius of one in both the planes. 1D HFLBP-TOP gives accuracy of 94.28 percentage result for DT recognition.

\section{CONCLUSION}

Rotation-Invariant local binary pattern method, LBP-HF and LBP-TOP outperform existing local binary patterns methods for static textures and Dynamic textures. We have implemented existing methods with code matrix and code file for static and dynamic textures respectively which contains uniform bin in a structured format which gives significantly faster performance as compare to traditional approach.

Our approach for Rotation invariant static textures gives 77.01 percentage classification results as compare to other LBP based methods on OUTEX_TC_00012. For dynamic texture it preformed good as compare to VLBP which has a drawback of bigger feature vector size. HFLBP-TOP gives 94.28 recognition rate over DynTex videos.

Future work for this is implementing LBP methods in cylindrical Co-ordinate system for rotation-invariant or as current world scenario is mainly based on time constraint so one can think to implement existing methodologies on multicore platform or on GPUs for faster classification.

\section{REFERENCES}

[1] Ojala, T., Pietikäinen, M.,: "Multiresolution Gray-scale and Rotation Invariant Texture Classification with Local Binary Pattern." IEEE Transactions on PAMI, vol. 24(7), 971-987 (2002)

[2] G. Zhao, T. Ahonen ,J. Matas and M. Pietikäine, "Rotation-Invariant Image and Video Description With LBP Features" IEEE Transactions on image process. Vol. 21, NO 4, April 2012

[3] Zhao, G., Pietikäinen, M.' 'Dynamic Texture Recognition Using Local Binary Patterns with an Application to Facial Expressions" IEEE Trans. PAMI, vol. 27(6), 915-928 (2007).

[4] T. Ahonen, J. Matas, C. He, and M. Pietikäinen, "Rotation invariant image description with local binary pattern histogram Fourier features,"in Proc. 16th Scand. Conf. Image Anal., 2009, pp. 2037-2041.

[5] Ahonen, T., Hadid, A., Pietikäinen, M., "Face Recognition with Local Binary Patterns: Application to Face Recognition". IEEE Trans. PAMI, vol. 28(12), 2037-2041 (2006)

[6] Guo, Z., Zhang, L., "A Completed Modeling of Local Binary Pattern Operator for Texture Classification" IEEE Trans. IP 19, 1657-1663 (2010).

[7] H. Arof and F. Deravi, "Circular neighbourhood and 1-D DFT features for texture classification and segmentation," Proc. Inst. Elect. Eng.-Vis., Image Signal Process., vol. 145, no. 3, pp. 167-172, Jun.1998. 